\title{
Graphene Induced Surface Reconstruction of $\mathrm{Cu}$
}

\author{
Jifa Tian, ${ }^{\dagger, \ddagger}$ Helin Cao, ${ }^{\dagger, \ddagger}$ Wei Wu, ${ }^{\S}$ Qingkai Yu, ${ }^{\|}$Nathan P. Guisinger, ${ }^{*, \perp}$ and Yong P. Chen ${ }^{*}, \dagger, \ddagger, \#$ \\ ${ }^{\dagger}$ Department of Physics, Purdue University, West Lafayette, Indiana 47907, United States \\ ${ }^{\ddagger}$ Birck Nanotechnology Center, Purdue University, West Lafayette, Indiana 47907, United States \\ ${ }^{\S}$ Center for Advanced Materials, and Department of Electrical and Computer Engineering, University of Houston, Houston, Texas \\ 77204, United States \\ "Ingram School of Engineering and Materials Science, Engineering and Commercialization Program, Texas State University, San \\ Marcos, Texas 78666, United States \\ ${ }^{\perp}$ Center for Nanoscale Materials, Argonne National Laboratory, Argonne, Illinois 60439, United States \\ ${ }^{\#}$ School of Electrical and Computer Engineering, Purdue University, West Lafayette, Indiana 47907, United States
}

\section{Supporting Information}

\begin{abstract}
An atomic-scale study utilizing scanning tunneling microscopy (STM) in ultrahigh vacuum (UHV) is performed on large single crystalline graphene grains synthesized on $\mathrm{Cu}$ foil by a chemical vapor deposition (CVD) method. After thermal annealing, we observe the presence of periodic surface depressions (stripe patterns) that exhibit long-range order formed in the area of $\mathrm{Cu}$ covered by graphene. We suggest that the observed stripe pattern is a $\mathrm{Cu}$ surface reconstruction formed by partial dislocations (which appeared to be stair-rod-like) resulting from the strain induced by the graphene overlayer. In addition, these graphene grains are shown to be more decoupled from the Cu substrate compared

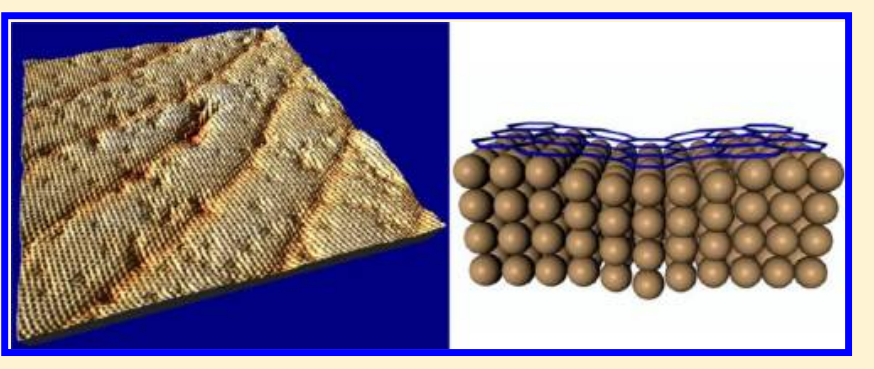
to previously studied grains that exhibited Moiré patterns.

KEYWORDS: Graphene, Cu, STM, strain, surface reconstruction, dislocation

$G$ raphene, one atomic layer of carbon organized in a honeycomb lattice, has exhibited spectacular electronic properties and stimulated intense research interest for applications in nanoelectronics. ${ }^{1,2}$ Since the pioneering work on exfoliated single layer graphene, numerous efforts have been made to advance both the techniques of growing graphene and the investigations of graphene properties. ${ }^{2-10}$ The successful synthesis of large-area graphene films ${ }^{5-10}$ has accelerated the potential applications of graphene in nanoelectronics and other technological areas. In the past few years, the chemical vapor deposition (CVD) growth technique has been applied to synthesize large-scale graphene on various metal surfaces. By this technique, a hydrocarbon gas ( such as $\mathrm{CH}_{4}$ ) is passed at high temperature over metal substrates, such as $\mathrm{Cu},{ }^{7-9} \mathrm{Ni}$, ${ }^{6,10,11}$ $\mathrm{Ru},{ }^{12-14}$ and $\mathrm{Ir}^{15-17}$ resulting in the formation of graphene layers on the surface. Among them, the $\mathrm{Cu}$ foil has become the most popular substrate due to its low cost and the fact that graphene films grown on it are predominantly monolayer and can be easily transferred to other substrates. ${ }^{7-9}$ On the other hand, grain boundaries that form in the continuous films limit their carrier mobility and induce carrier scattering that could degrade the quality of graphene devices. ${ }^{18-20}$ Recently, we synthesized and characterized hexagonally shaped single crystalline graphene grains on $\mathrm{Cu}$ foils grown by $\mathrm{CVD}$ techniques. ${ }^{18,19}$ Here, we further study the interaction between graphene grains and underlying $\mathrm{Cu}$ foil substrate at the atomic- scale using scanning tunneling microscopy (STM). Such a study may also help us understand the graphene growth mechanism and improve the quality of the CVD graphene films.

STM has been widely used to probe the atomic-scale structure and electronic properties (such as local density of states, LDOS) of graphene films grown on silicon carbide, ${ }^{5,21}$ metal substrates, ${ }^{12,14,22,23}$ and exfoliated graphene on $\mathrm{SiO}_{2} /$ $\mathrm{Si}^{24-26}$ In our work, the STM has been utilized to study graphene grains grown on polycrystalline $\mathrm{Cu}$ foil. Particular efforts are dedicated to study the interaction between graphene and underlying $\mathrm{Cu}$ substrate. The STM experiments are performed on the as-grown grains transferred into ultrahigh vacuum (UHV) followed by annealing at $400{ }^{\circ} \mathrm{C}$. Our investigation reveals the presence of well-ordered stripe patterns with $\sim 5 \mathrm{~nm}$ periodicity confined to the regions of large-area graphene grains. These stripes are interpreted as a reconstruction of $\mathrm{Cu}$ surface due to partial dislocations formed beneath the graphene in the surface of the $\mathrm{Cu}$ to relax the tensile strain induced by the graphene overlayer. Furthermore, we find that these graphene grains are more decoupled from

Received: January 24, 2012

Revised: July 6, 2012

Published: July 17, 2012 


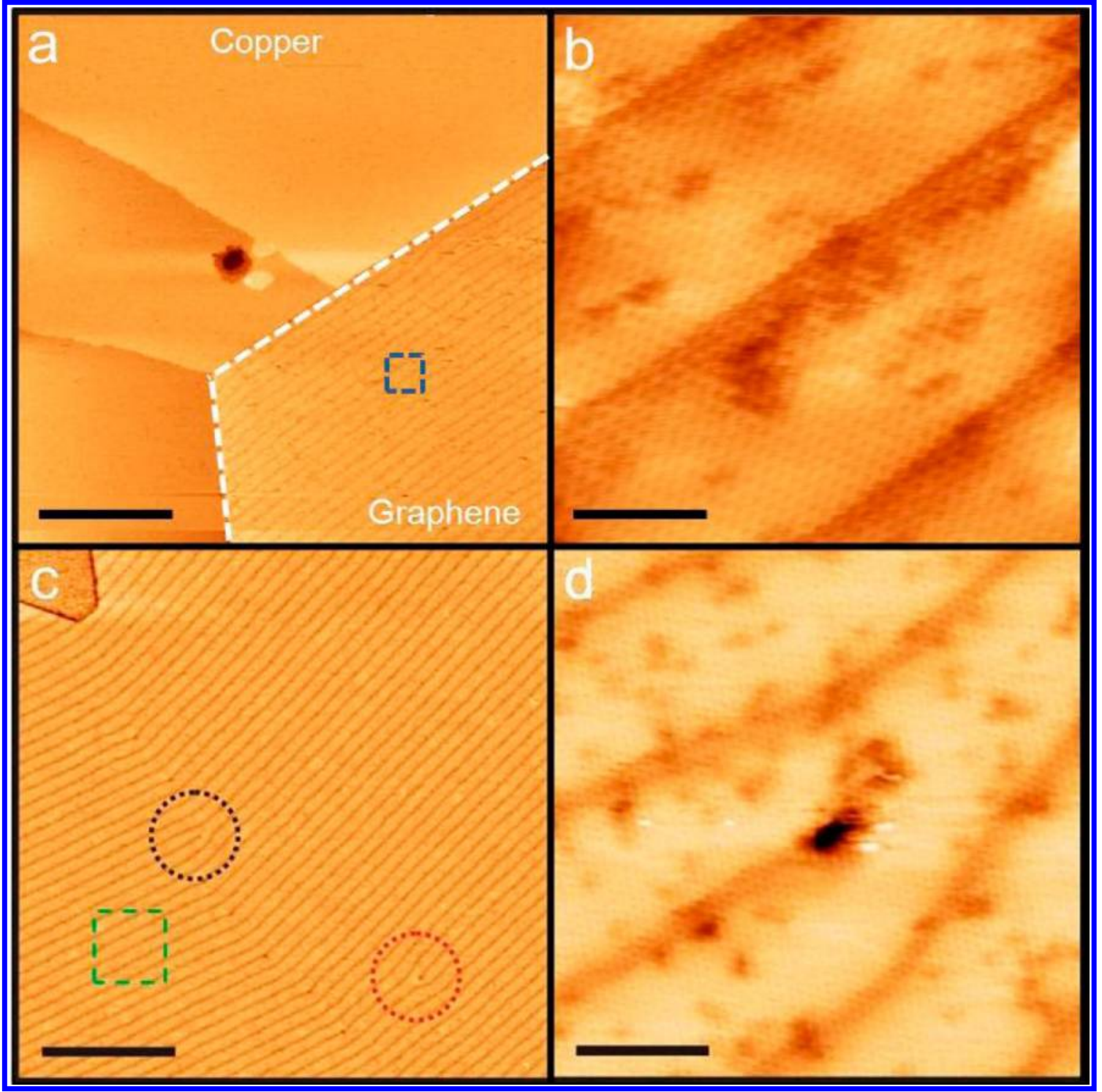

Figure 1. STM images of graphene grains on $\mathrm{Cu}$ foil: (a) An STM topographic image of a graphene grain after annealing at $400{ }^{\circ} \mathrm{C}$ for $48 \mathrm{~h}$. The scale bar is $40 \mathrm{~nm}$. Measurement conditions: tunneling current $I=100 \mathrm{pA}$, sample-tip bias voltage $V=-200 \mathrm{mV}$. (b) The zoomed-in atomicresolved STM topographic image taken from the blue dashed box in a (measurement conditions: $I=20 \mathrm{nA}, V=-200 \mathrm{mV}$ ). The scale bar is $2 \mathrm{~nm}$. (c) Another low magnification STM topographic image of the stripe patterns and graphene grain (measurement conditions: $I=1 \mathrm{nA}, V=-200$ $\mathrm{mV}$ ). The dotted circles (in black and red) indicate locations where new lines are initiated in the stripe patterns. The scale bar is $50 \mathrm{~nm}$. (d) The zoomed-in STM topographic image from the area indicated by a dotted black circle in c. The scale bar is $5 \mathrm{~nm}(I=20 \mathrm{nA}, V=-200 \mathrm{mV})$.

the substrate compared to those studied in several previous reports $^{22,27}$ (where the graphene domains are much smaller than ours, did not induce dislocations in the underlying $\mathrm{Cu}$, and exhibited Moiré patterns).

The graphene samples used in this work are synthesized on polycrystalline $\mathrm{Cu}$ foils (25- $\mu$ m-thick, $99.8 \%$, Alfa Aesar) by ambient $\mathrm{CVD}\left(\mathrm{CH}_{4}\right.$ as carbon stock), using similar procedures as in our previous publications. ${ }^{18,28}$ The growth is carried out at $1050{ }^{\circ} \mathrm{C}$ and stopped before the graphene grains would have merged with each other to form a globally continuous ${ }^{9,29}$ (but polycrystalline) graphene film. Then, the sample is rapidly cooled to room temperature under the protection of $\mathrm{Ar}$ and $\mathrm{H}_{2}$. Finally, the samples are removed from the CVD tube furnace (thus exposed to air) before being transferred into the UHV STM chamber.

The STM measurements are carried out at room temperature in an Omicron STM with a base pressure $<10^{-11} \mathrm{mbar}$, with electrochemically etched STM tips made of tungsten or platinum/iridium alloy. After the sample is moved into the UHV chamber, it is annealed at $400{ }^{\circ} \mathrm{C}$ for $48 \mathrm{~h}$. We perform both STM topography as well as scanning tunneling spectroscopy (STS) measurements. The STS and differential conductance $(\mathrm{d} I / \mathrm{d} V)$ are measured with lock-in detection by applying a small modulation ( $30 \mathrm{mV} \mathrm{rms}$ in amplitude) to the tunneling voltage at $\sim 10 \mathrm{kHz}$.

The typical shape of a graphene grain is hexagonal, ${ }^{18}$ and in some instances we observe merged multiple hexagonal grains (Figure S1a of the Supporting Information, showing an SEM image after taking out of CVD furnace, but before UHV thermal annealing). A morphological difference between bare $\mathrm{Cu}$ and graphene grains can be clearly observed (see also Figure S1b, showing the STM image of a typical unannealed sample). A significant source of the observed roughness on graphene is attributed to the steps on the underlying $\mathrm{Cu}$ substrate, ${ }^{18}$ giving 


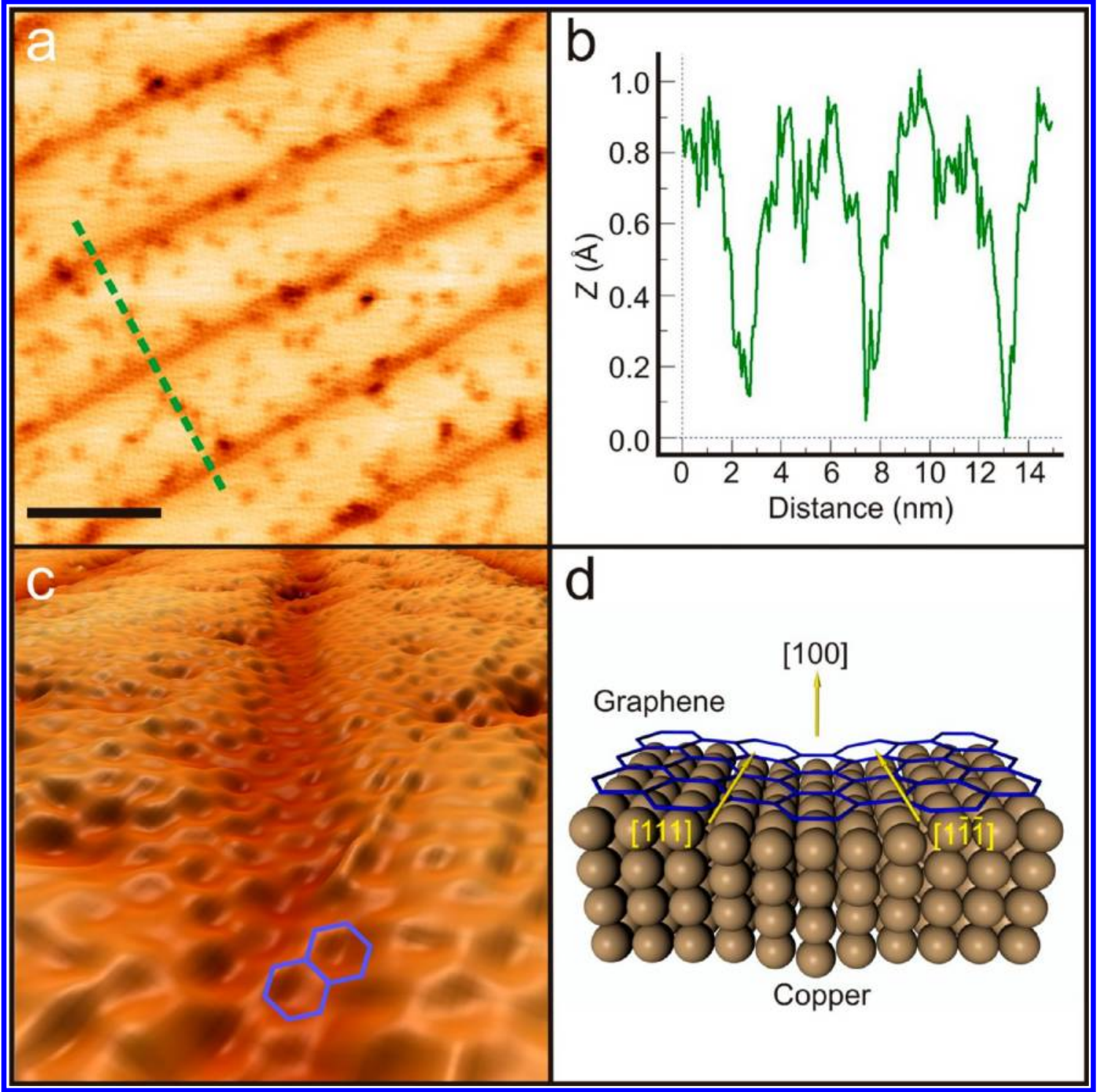

Figure 2. Characterization and interpretation of the observed stripe pattern as reconstruction due to partial dislocations on Cu surface underneath graphene: (a) An STM topographic image from the area indicated by green dashed box in Figure 1c, showing surface depressions (interpreted as partial dislocation lines on $\mathrm{Cu})$ under graphene lattice. The scale bar is $6 \mathrm{~nm}(I=20 \mathrm{nA}, V=-200 \mathrm{mV})$. (b) The height profile along the green dashed line shown in a. (c) Three dimensional (3D) rendition of a high resolution STM topographic image acquired near a typical dislocation line (I $=20 \mathrm{nA}, V=-200 \mathrm{mV}$ ). (d) Schematic model (not to scale) of a stair-rod-like dislocation on Cu (with surface orientation assumed to be (100) in this example) underneath graphene grain.

rise to the line texture seen in Figure S1a,b. These line textures are nonuniform with spacing over hundreds of nanometers and height differences of $\sim 10 \mathrm{~nm}^{18}$ Such steps are nearly unobservable on bare $\mathrm{Cu}$ surface not covered (and protected) by graphene, due to the formation of a thin layer of native copper oxide. ${ }^{18,27,30}$ These results reveal that graphene appears to be an excellent impermeable barrier against oxidation or other forms of surface chemistry on $\mathrm{Cu}$. Our previous STM study demonstrated that high temperature annealing can help remove such $\mathrm{Cu}$ oxide. ${ }^{27}$

Once introduced into the UHV STM system, the sample is annealed at $400{ }^{\circ} \mathrm{C}$ for $48 \mathrm{~h}$, following which large single crystalline graphene grains are located, as illustrated in the STM topographic image of Figure 1a. On the $\mathrm{Cu}$ surface outside graphene, the oxide can be partially removed by this thermal annealing process. From the STM topographic image (Figures 1a, compared to the unannealed samples, Figure S1a,b of the
Supporting Information), we can see that the post-annealing $\mathrm{Cu}$ surface outside graphene becomes much smoother and shows up the $\mathrm{Cu}$ atomic steps. Most strikingly, we find that the surface morphology within the graphene grain has also changed dramatically after the annealing, where a long-range periodic stripe pattern (appearing as many periodic dark lines largely parallel to one of the edges of the graphene grain in the topographic image Figure 1a) is observed. Figure $1 \mathrm{~b}$ further shows a few such dark lines in a zoomed-in atomically resolved topographic image taken from the area indicated by the blue dashed box in Figure 1a. There are no apparent distortions, defects, or electronic scattering within the graphene lattice at the dark lines (see also Figure S2). This stripe pattern, which will be interpreted as a graphene-induced $\mathrm{Cu}$ surface reconstruction, is the main finding of this paper.

Figure 1c shows another example of such a stripe pattern observed in the graphene region. The stripes again appear in 
the topographic image as uniformly spaced dark lines, corresponding to depressions in the surface. Two additional interesting features are observed in this case. One is that within this graphene region the orientation of the stripes is found to change by $\sim 18^{\circ}$, as seen in Figure 1c from the upper-right to the lower-left of the image. Taking a Fourier transform (FT) of this image, we can see that the stripe pattern gives rise to two sets of spots (indicated by green and red circles in Figure S3 of the Supporting Information, respectively). The angle between the two sets of spots corresponds to the orientation change $\left(\sim 18^{\circ}\right)$ of the stripe pattern. Another interesting observation is the two new dark lines initiating from the locations highlighted by the dashed black and red circles in Figure 1c near the transition region between the two orientations. Figure 1d shows a high magnification STM image for the stripe pattern taken from the region highlighted by the dashed black circle in Figure 1c. Importantly, it can be seen that the graphene lattice remains coherent (single crystalline) within the entire region, even around where the orientation of the stripes changes or the new dark line initiates.

An atomically resolved STM image and the corresponding height profile of a zoomed-in region containing several such well-ordered stripes (dark lines, indicating surface depressions) are shown in Figure 2a,b, respectively. It can be seen that the spacings of these dark lines are uniform, with a periodicity $\sim 5$ $\mathrm{nm}$. The depth of the depression measured on graphene surface is $\sim 0.6 \AA$ (Figure $2 \mathrm{~b}$ ). Figure $2 \mathrm{c}$ gives the three-dimensional (3D) rendition of a high resolution STM topographic image acquired around one of such lines. We can again clearly see the graphene lattice highlighted by the blue hexagons remains intact crossing the dark line.

While in principle both wrinkles (ripples) on graphene $e^{31,32}$ as well as Moiré patterns ${ }^{27,33}$ between graphene and $\mathrm{Cu}$ could give rise to line patterns, neither of those is consistent with the features of the stripe pattern we have observed. The graphene wrinkles or ripples typically appear as surface protrusions, ${ }^{31,32}$ not depressions. They are also more irregularly spaced, with much larger sizes and separations than our dark lines. ${ }^{31,32}$ The periodicity of the Moiré pattern is sensitive to its orientation, $^{27,33}$ whereas our stripe pattern maintains its periodicity even with an orientation change (Figure 1c). Furthermore, the dark lines in our stripe pattern are not perfectly straight and can also show occasional bending or curvatures (as seen in Figures 1a,c and 2a), unlike the highly straight lines that would be produced by a lattice superstructure from the Moire pattern. ${ }^{27,33}$ Therefore we conclude that the dark lines (which appear to minimally perturb the graphene lattice) of our stripe pattern most likely arise from features (in this case depressions) formed on the $\mathrm{Cu}$ surface underneath the graphene. Since they only appear under graphene after appropriate thermal annealing, they represent a grapheneinduced reconstruction of $\mathrm{Cu}$ surface, and we speculate that they result from partial dislocations due to the strain between graphene and $\mathrm{Cu}$, as discussed below.

It is well-known that the $\mathrm{Cu}$ surface and adsorbate-covered $\mathrm{Cu}$ surface can reconstruct. ${ }^{34-38}$ Previous studies on $\mathrm{Cu}$ covered by adatoms further showed that such surface reconstruction can be quite sensitive to thermal annealing as well as adatom coverage. ${ }^{34,35}$ The reconstruction feature sizes and spacings are on the similar orders of magnitude as those in our observation. In addition, lattice reconstructions due to formation of partial dislocations ${ }^{39}$ to release the strain are common in many metal surfaces or heteroepitaxial thin films, as have been reported in numerous systems such as $\mathrm{Au}(111),{ }^{40,41}$ $\mathrm{Ni} / \mathrm{Cu}(001),{ }^{42} \mathrm{Ni} / \mathrm{Ir}(100),{ }^{39} \mathrm{Co} / \operatorname{Ir}(100),{ }^{39} \mathrm{Cu} / \mathrm{Ru}(0001),{ }^{43}$ and so forth. In our case, although the CVD growth of graphene on $\mathrm{Cu}$ foil may not be epitaxial, ${ }^{18}$ strain can still be induced after growth at high temperature followed by cooling down to room temperature, during which the graphene lattice can expand (due to its negative coefficient of thermal expansion ${ }^{6-8,44}$ ) while $\mathrm{Cu}$ shrinks, giving rise to a tensile strain on $\mathrm{Cu}$. During the subsequent thermal annealing in $\mathrm{UHV}$, the top few atomic layers of $\mathrm{Cu}$ underneath graphene may become unstable and start to reconstruct, forming (periodic) depressions on the $\mathrm{Cu}$ surface thus releasing the tensile strain. A possible mechanism for such reconstruction of the top few atomic layers of $\mathrm{Cu}$ underneath graphene is through the formation of partial dislocations. The partial dislocations can give rise to the long-range ordered depressions/stripes in $\mathrm{Cu}$ shown as the dark lines in the STM topographic images. We note that such dark lines (depressions) are reminiscent of stair-rod dislocations (a common form of surface misfit partial dislocations) previously found in heteroepitaxial thin films. ${ }^{39}$ It is also worth noting that in our case the partial dislocations are formed in the surface layers of the bulk substrate $(\mathrm{Cu})$ instead of the overlayer (graphene). Indeed the graphene lattice appears to be intact with no signs of reconstruction in our STM images (Figures $1 \mathrm{~b}, \mathrm{~d}, 2 \mathrm{a}, \mathrm{c}$, and S2). This is a novel situation and very different from the case observed in the growth of many heteroepitaxial thin films $\mathrm{s}^{39,42,43}$ where the dislocations are typically formed in the overlayer (heteroepitaxial thin films). We believe this difference reflects the high mechanical strength and stability of graphene lattice, ${ }^{45}$ making the reconstruction happen in $\mathrm{Cu}$ rather than graphene during thermal annealing.

Figure 2d displays a proposed schematic model for an example of the stair-rod-like ${ }^{39}$ dislocation in $\mathrm{Cu}$ under graphene. The model is presented for a $\mathrm{Cu}$ surface orientation of (100). Even though we do not have a direct measurement of the local $\mathrm{Cu}$ surface orientation under the stripe pattern, (100) has been found to be the predominant surface orientation (with no other orientations detected) for our $\mathrm{Cu}$ foil from our X-ray diffraction measurements, ${ }^{27}$ as well as the linear Moiré pattern $^{27}$ (see also Figure S4 of the Supporting Information and discussions below) observed from smaller and further annealed graphene grains on the same $\mathrm{Cu}$ foil. The principal mechanism for the formation of such a stair-rod dislocation 39 can be understood as the interaction of two partial dislocations taking place at the $\mathrm{Cu}(111)$ and $(1 \overline{11})$ faces (resulting from the refaceting of the $\mathrm{Cu}$ (100) surface under tensile strain). The result of the interaction of the two dislocations, which meet at their apex and eventually form a stair-rod-like dislocation, is displayed in Figure 2d. We emphasize that the proposed mechanism and microstructure for the observed reconstruction as stair-rod-like dislocations are speculative at this point, and need confirmation by further experimental (including additional probes such as LEED (low energy electron diffraction)) as well as theoretical studies. More work is also needed to better understand the physical conditions for the reconstruction to occur, what sets its orientation and periodicity, and whether qualitatively similar surface reconstructions can occur generically on $\mathrm{Cu}$ surfaces with different orientations.

The long-range periodic stripe pattern associated with graphene-induced $\mathrm{Cu}$ surface reconstruction in this work has not been seen in previous STM studies on unannealed or low temperature $\left(300{ }^{\circ} \mathrm{C}\right)$ annealed graphene grains on $\mathrm{Cu}$ foils ${ }^{18}$ 


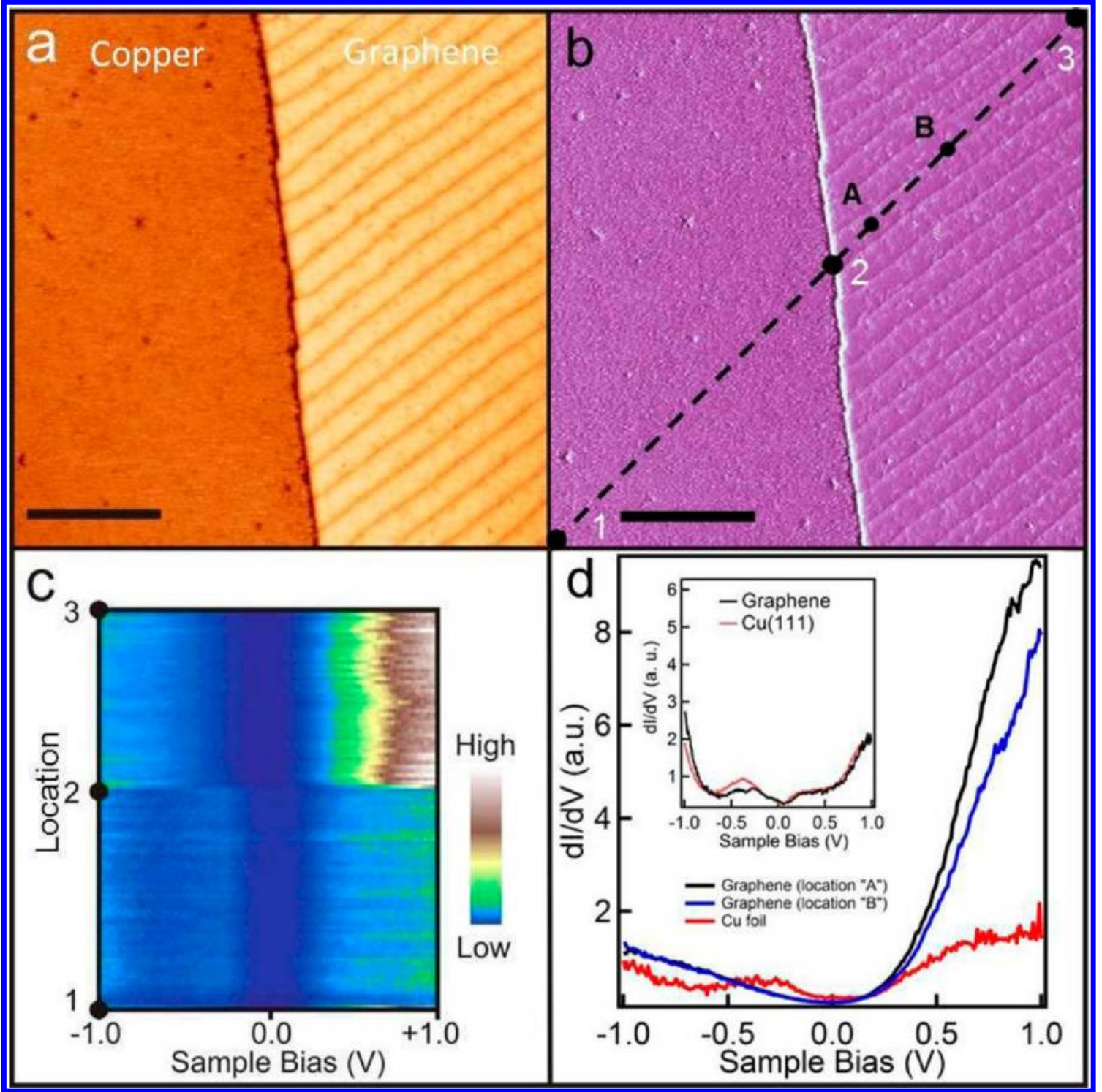

Figure 3. STM image and scanning tunneling spectroscopy (STS) of a graphene grain on Cu foil. (a) STM topographic image showing both graphene and $\mathrm{Cu}$ foil. (b) Differential conductance $\mathrm{d} I / \mathrm{d} V$ map recorded simultaneously with the topographic image (a). For a, b, the scale bar is 25 $\mathrm{nm}(I=1 \mathrm{nA}, V=-200 \mathrm{mV})$. (c) $\mathrm{d} I / \mathrm{d} V$ spectra recorded at 100 points in equal distance along the black line in b with numbers " 1 ", "2", and " 3 " indicating three representative locations labeled in b. (d) Representative $\mathrm{d} I / \mathrm{d} V$ spectra recorded inside (at a two representative locations " $\mathrm{A}$ " and "B" in b) and outside a graphene grain. The inset (reproduced from ref 22) shows $\mathrm{d} I / \mathrm{d} V$ spectra in a previously study ${ }^{22}$ of nm-sized graphene islands on $\mathrm{Cu}(111)$.

or on small graphene grains (on single crystalline $\mathrm{Cu}^{22}$ or $\mathrm{Cu}$ foils ${ }^{27}$ ) after further annealing or thermal cycling at much higher temperatures than ours. It is possible that the $\mathrm{Cu}$ surface reconstruction (partial dislocations) we observed could be dependent on various factors such as the thermal annealing or processing condition (as found to be the case previously for $\mathrm{Cu}$ surface with adsorbates ${ }^{34,35}$ ), the size of graphene grains (as smaller grains may be able to release the strain more easily), and other conditions of $\mathrm{Cu}$ substrate (eg., impurities) underneath the graphene grain, and so forth. In addition, the previous work in ref 27 (which studied much-smaller-sized, further annealed graphene grains on the same $\mathrm{Cu}$ foil as in our current work) observed clear Moiré patterns (formed between graphene and $\mathrm{Cu}$ (100) lattices). No Moiré patterns are observed in our (larger size and less annealed) graphene grains. We believe this absence of Moiré patterns reflects a weaker electronic coupling between our graphene grain and $\mathrm{Cu}$ substrate than that in ref 27 . This is also consistent with the much larger apparent graphene- $\mathrm{Cu}$ height difference we observe for our graphene grain than for smaller, furtherannealed grains similar to ref 27 (Figure S4, Supporting Information).

We have further characterized the electronic properties of our graphene grains using STS spectroscopy and mapping. ${ }^{22}$ Figure $3 \mathrm{~b}$ is a differential conductance $\mathrm{d} I / \mathrm{d} V$ map (at a sample bias of $-200 \mathrm{mV}$ ) measured concurrently with the topographic image Figure 3a and shows a higher LDOS over the graphene grain compared to the outside $\mathrm{Cu}$. This electronic contrast allows us to easily identify the two materials when imaging large areas. We have also performed individual $\mathrm{d} I / \mathrm{d} V$ spectral measurements at 100 points along a line (the black dashed line in Figure $3 \mathrm{~b}$ with numbers "1", " 2 ", and " 3 " highlighting a few representative points). The collection of 100 spectra is shown as a $2 \mathrm{D}$ color plot in Figure $3 \mathrm{c}$. It is worth noting that the 
transition between the electronic properties of the graphene (between " 2 " and " 3 ") and Cu regions (between " 1 " and " 2 ") is very abrupt, while the spectra taken within the same region (either graphene or $\mathrm{Cu}$ ) are similar. Three individual spectra from this measurement are plotted in Figure 3d, where the red curve is measured over the $\mathrm{Cu}$ surface outside the graphene grain and the black and blue curves inside the graphene grain (measured from locations "A" and "B" in Figure $3 \mathrm{~b}$, on and away from a $\mathrm{Cu}$ dislocation line, respectively). There is no significant difference between the $\mathrm{d} I / \mathrm{d} V$ spectra measured from "A" and "B", except for a slightly lower value of $\mathrm{d} I / \mathrm{d} V$ in the spectrum " $\mathrm{B}$ " on the positive bias side, affirming that the surface depressions (dark lines) on $\mathrm{Cu}$ do not appreciably perturb the electronic properties of the graphene overlayer. The "bump" feature in the negative bias side measured in $\mathrm{Cu}$ spectrum (observed to be even more prominent on bare $\mathrm{Cu}$ foil more extensively annealed with most of the native oxide removed, Figure S5 of the Supporting Information) is not seen in the graphene spectrum, again consistent with the relatively weak electronic coupling between our graphene gain and $\mathrm{Cu}$ (whereas in a previous study ${ }^{22}$ of graphene grains strongly coupled to $\mathrm{Cu}$ and exhibiting Moiré patterns, the graphene can be nearly "electronically" transparent with similar $\mathrm{d} I / \mathrm{d} V$ spectrum as $\mathrm{Cu}$, as shown in the inset, which is reproduced from Figure 3c of ref 22).

In summary, we have conducted atomically resolved topographic and spectroscopic investigations of the graphene grains on $\mathrm{Cu}$ foil by STM. After annealing at $400{ }^{\circ} \mathrm{C}$ for $48 \mathrm{~h}$, the $\mathrm{Cu}$ surface underneath the graphene grain forms periodic stripe patterns, which we associate with a reconstruction of the $\mathrm{Cu}$ surface due to the formation of partial dislocations (such as stair-rod-like dislocations) to release the strain induced by graphene. The graphene lattice remains intact without defects when it crosses the dislocations underneath. No Moiré patterns are observed in graphene and the coupling between our graphene grain, and the $\mathrm{Cu}$ substrate appears to be relatively weak. Our findings may shed light on the interaction between $\mathrm{Cu}$ and graphene as well as the growth mechanism of graphene on $\mathrm{Cu}$ foils.

\section{ASSOCIATED CONTENT}

\section{S Supporting Information}

SEM and topographic STM images of unannealed graphene grains, additional atomically resolved STM images, the corresponding $\mathrm{d} I / \mathrm{d} V$ maps and FFT image, comparing topographic STM images of graphene grains under different thermal annealing, and the differential conductance $(\mathrm{d} I / \mathrm{d} V)$ spectrum measured from a bare $\mathrm{Cu}$ foil that has gone through extensive UHV thermal annealing. This material is available free of charge via the Internet at http://pubs.acs.org.

\section{AUTHOR INFORMATION}

\section{Corresponding Author}

*E-mail: nguisinger@anl.gov and yongchen@purdue.edu.

\section{Notes}

The authors declare no competing financial interest.

\section{ACKNOWLEDGMENTS}

This work was performed under the auspices of Argonne National Laboratory (ANL) Center for Nanoscale Materials (CNM) User Research Program (Proposal ID 998) and partially supported by the NSF, DHS and NRI-MIND center.
The user facilities at ANL's CNM are supported by the U.S. Department of Energy, Office of Science, Office of Basic Energy Sciences, under Contract No. DE-AC02-06CH11357. N.P.G. acknowledges DOE "SISGR" Contract No. DE-FG0209ER16109. The authors also thank Prof. C. K. Shih for valuable discussions.

\section{REFERENCES}

(1) Castro Neto, A. H.; Guinea, F.; Peres, N. M. R.; Novoselov, K. S.; Geim, A. K. Rev. Mod. Phys. 2009, 81, 109-162.

(2) Geim, A. K.; Novoselov, K. S. Nat. Mater. 2007, 6, 183-191.

(3) Novoselov, K. S.; Geim, A. K.; Morozov, S. V.; Jiang, D.; Zhang, Y.; Dubonos, S. V.; Grigorieva, I. V.; Firsov, A. A. Science 2004, 306, 666-669.

(4) Zhang, Y.; Tan, Y.-W.; Stormer, H. L.; Kim, P. Nature 2005, 438, 201-204.

(5) Berger, C.; Song, Z.; Li, X.; Wu, X.; Brown, N.; Naud, C.; Mayou, D.; Li, T.; Hass, J.; Marchenkov, A. N.; Conrad, E. H.; First, P. N.; de Heer, W. A. Science 2006, 312, 1191-1196.

(6) Kim, K. S.; Zhao, Y.; Jang, H.; Lee, S. Y.; Kim, J. M.; Kim, K. S.; Ahn, J. H.; Kim, P.; Choi, J. Y.; Hong, B. H. Nature 2009, 457, 706710 .

(7) Li, X.; Cai, W.; An, J.; Kim, S.; Nah, J.; Yang, D.; Piner, R.; Velamakanni, A.; Jung, I.; Tutuc, E.; Banerjee, S. K.; Colombo, L.; Ruoff, R. S. Science 2009, 324, 1312-1314.

(8) Bae, S.; Kim, H.; Lee, Y.; Xu, X.; Park, J.-S.; Zheng, Y.; Balakrishnan, J.; Lei, T.; Kim, H. R.; Song, Y. I.; Kim, Y.-J.; Kim, K. S.; Özyilmaz, B.; Ahn, J.-H.; Hong, B. H.; Iijima, S. Nat. Nanotechnol. 2010, 5, 574-578.

(9) Cao, H.; Yu, Q.; Jauregui, L.; Tian, J.; Wu, W.; Liu, Z. H.; Jalilian, R.; Benjamin, D. K.; Jiang, Z. G.; Bao, J. M.; Pei, S. S.; Chen, Y. P. Appl. Phys. Lett. 2010, 96, No. 122106.

(10) Reina, A.; Jia, X.; Ho, J.; Nezich, D.; Son, H.; Bulovic, V.; Dresselhaus, M. S.; Kong, J. Nano Lett. 2009, 9, 30-35.

(11) Park, H. J.; Meyer, J.; Roth, S.; Skakalova, V. Carbon 2010, 48, 1088-1094.

(12) Martoccia, D.; Willmott, P. R.; Brugger, T.; Bjorck, M.; Gunther, S.; Schleputz, C. M.; Cervellino, A.; Pauli, S. A.; Patterson, B. D.; Marchini, S.; Wintterlin, J.; Moritz, W.; Greber, T. Phys. Rev. Lett. 2008, 101, No. 126102.

(13) Sutter, P. W.; Flege, J.-I.; Sutter, E. A. Nat. Mater. 2008, 7, 406411.

(14) Pan, Y.; Zhang, H. G.; Shi, D. X.; Sun, J. T.; Du, S. X.; Liu, F.; Gao, H.-J. Adv. Mater. 2009, 21, 2777-2780.

(15) Coraux, J.; N’Diaye, A. T.; Busse, C.; Michely, T. Nano Lett. 2008, 8, 565-570.

(16) Lacovig, P.; Pozzo, M.; Alfe, D.; Vilmercati, P.; Baraldi, A.; Lizzit, S. Phys. Rev. Lett. 2009, 103, No. 179904.

(17) Balog, R.; Jørgensen, B.; Nilsson, L.; Andersen, M.; Rienks, E.; Bianchi, M.; Fanetti, M.; Lægsgaard, E.; Baraldi, A.; Lizzit, S.; Sljivancanin, Z.; Besenbacher, F.; Hammer, B.; Pedersen, T. G.; Hofmann, P.; Hornekær, L. Nat. Mater. 2010, 9, 315-319.

(18) Yu, Q.; Jauregui, L. A.; Wu, W.; Colby, R.; Tian, J.; Su, Z.; Cao, H.; Liu, Z.; Pandey, D.; Wei, D.; Chung, T. F.; Peng, P.; Guisinger, N. P.; Stach, E. A.; Bao, J.; Pei, S.-S.; Chen, Y. P. Nat. Mater. 2011, 10, 443-449.

(19) Jauregui, L. A.; Cao, H.; Wu, W.; Yu, Q.; Chen, Y. P. Solid State Commun. 2011, 151, 1100-1104.

(20) Li, X.; Magnuson, C. W.; Venugopal, A.; An, J.; Suk, J. W.; Han, B.; Borysiak, M.; Cai, W.; Velamakanni, A.; Zhu, Y.; Fu, L.; Vogel, E. M.; Voelkl, E.; Colombo, L.; Ruoff, R. S. Nano Lett. 2010, 10, 43284334.

(21) Rutter, G. M.; Crain, J. N.; Guisinger, N. P.; Li, T.; First, P. N.; Stroscio, J. A. Science 2007, 317, 219-222.

(22) Gao, L.; Guest, J. R.; Guisinger, N. P. Nano Lett. 2010, 10, $3512-3516$. 
(23) Eom, D.; Prezzi, D.; Rim, K. T.; Zhou, H.; Lefenfeld, M.; Xiao, S. X.; Nuckolls, C.; Hybertsen, M. S.; Heinz, T. F.; Flynn, G. W. Nano Lett. 2009, 9, 2844-2848.

(24) Stolyarova, E.; Rim, K. T.; Ryu, S.; Maultzsch, J.; Kim, P.; Brus, L. E.; Heinz, T. F.; Hybertsen, M. S.; Flynn, G. W. Proc. Natl. Acad. Sci. U.S.A. 2007, 104, 9209-9212.

(25) Ishigami, M.; Chen, J. H.; Cullen, W. G.; Fuhrer, M. S.; Williams, E. D. Nano Lett. 2007, 7, 1643-1648.

(26) Zhang, Y.; Brar, V. W.; Girit, C.; Zettl, A.; Crommie, M. F. Nat. Phys. 2009, 5, 722-726.

(27) Cho, J.; Gao, L.; Tian, J.; Cao, H.; Wu, W.; Yu, Q.; Yitamben, E. N.; Fisher, B.; Guest, J. R.; Chen, Y. P.; Guisinger, N. P. ACS Nano 2011, 5, 3607-2613.

(28) Tian, J.; Cao, H.; Wu, W.; Yu, Q.; Chen, Y. P. Nano Lett. 2011, $11,3663-3668$.

(29) Wu, W.; Liu, Z. H.; Jauregui, L. A.; Yu, Q.; Pillai, R.; Cao, H.; Bao, J. M.; Chen, Y. P.; Pei, S. S. Sens. Actuators, B 2010, 150, 296300.

(30) Chen, S.; Brown, L.; Levendorf, M.; Cai, W.; Ju, S.-Y.; Edgeworth, J.; Li, X.; Magnuson, C. W.; Velamakanni, A.; Piner, R. D.; Kang, J.; Park, J.; Ruoff, R. S. ACS Nano 2011, 5, 1321-1327.

(31) Zhang, Y.; Gao, T.; Gao, Y.; Xie, S.; Ji, Q.; Yan, K.; Peng, H.; Liu, Z. ACS Nano 2011, 5, 4014-4022.

(32) Ni, G.-X.; Zheng, Y.; Bae, S.; Kim, H. R.; Pachoud, A.; Kim, Y. S.; Tan, C.-L.; Im, D.; Ahn, J.-H.; Hong, B. H.; Özyilmaz, B. ACS Nano 2012, 6, 1158-1164.

(33) Zhao, L.; Rim, K. T.; Zhou, H.; He, R.; Heinz, T. F.; Pinczuk, A.; Flynn, G. W.; Pasupathy, A. N. Solid State Commun. 2011, 151, 509-513.

(34) Guillemot, L.; Bobrov, K. Phys. Rev. B 2011, 83, No.075409.

(35) Kern, K.; Niehus, H.; Schatz, A.; Zeppendeld, P.; Goerge, J.; Comsa, G. Phys. Rev. Lett. 1991, 67, 855-858.

(36) Driver, S. M.; Hoeft, J.-T.; Plocik, M.; Kittel, M.; Rerborg, R.; Tooms, R. L.; Kang, J.-H.; Woodruff, D. P. J. Phys.: Condens. Matter 2001, 13, L601-L606.

(37) Harrison, M. J.; Woodruff, D. P.; Robinson, J. Phys. Rev. B 2006, 74 , No. 165402

(38) Jensen, F.; Besenbacher, F.; Laesgaard, E.; Stensgaard, I. Phys. Rev. B 1990, 41, No. 10233 .

(39) Klein, A.; Meyer, W.; Schmidt, A.; Gumler, B.; Muller, S.; Hammer, L.; Heinz, K. Phys. Rev. B 2008, 78, No. 045422.

(40) Wohl, C.; Chiang, S.; Wilson, R. J.; Lippel, P. H. Phys. Rev. B 1989, 39, 7988-7991.

(41) Barth, J. V.; Brune, H.; Ertl, G.; Behm, R. J. Phys. Rev. B 1990, 42, 9307-9318.

(42) Matthews, J. W.; Crawford, J. L. Thin Solid Films 1970, 5, 187198.

(43) Günther, C.; Vrijmoeth, J.; Hwang, R. Q.; Behm, R. J. Phys. Rev. Lett. 1995, 74, 754-757.

(44) Bao, W.; Miao, F.; Chen, Z.; Zhang, H.; Jang, W.; Dames, C.; Lau, C. N. Nat. Nanotechnol. 2009, 4, 562-566.

(45) Lee, C.; Wei, X.; Kysar, J. W.; Hone, J. Science 2008, 321, 385388. 